\title{
Ribosome-associated chaperones as key players in proteostasis
}

\author{
Steffen Preissler ${ }^{1,2}$ and Elke Deuerling ${ }^{1}$ \\ ${ }^{1}$ Molecular Microbiology, Department of Biology, University of Konstanz, 78457 Konstanz, Germany \\ ${ }^{2}$ Konstanz Research School Chemical Biology, University of Konstanz, 78457 Konstanz, Germany
}

\begin{abstract}
De novo protein folding is delicate and error-prone and requires the guidance of molecular chaperones. Besides cytosolic and organelle-specific chaperones, cells have evolved ribosome-associated chaperones that support early folding events and prevent misfolding and aggregation. This class of chaperones includes the bacterial trigger factor (TF), the archaeal and eukaryotic nascent polypeptide-associated complex (NAC) and specialized eukaryotic heat shock protein (Hsp) $70 / 40$ chaperones. This review focuses on the cellular activities of ribosome-associated chaperones and highlights new findings indicating additional functions beyond de novo folding. These activities include the assembly of oligomeric complexes, such as ribosomes, modulation of translation and targeting of proteins.
\end{abstract}

\section{Protein biogenesis is supported by a robust chaperone network}

Ribosomes synthesize proteins based on the genetic information delivered by mRNAs. After synthesis of the first 3540 amino acids, nascent chains exit the $\sim 100$ - $\AA$-long ribosomal tunnel. At that stage, multiple factors that directly bind to ribosomes interact with the emerging nascent polypeptides. Besides chaperones, processing enzymes cotranslationally modify the $\mathrm{N}$ termini of a subset of nascent peptides. In addition, targeting factors such as the signal recognition particle (SRP) initiate the delivery of secreted polypeptides to the membrane for translocation [1].

Folding of nascent proteins can start cotranslationally as the polypeptide exits the ribosomal tunnel, but it is only completed when the entire sequence is available on release from the ribosome. The ribosomal tunnel has a diameter between 10 and $20 \AA$ and therefore it allows the passage of unfolded polypeptides or of peptides in an $\alpha$-helical conformation but largely prevents the formation of structural elements involving long-distance interactions within the peptide chain [2]. Moreover, de novo protein folding is challenging because during synthesis, the sequence information of a nascent protein is incomplete and continuously changing; during elongation, approximately four and 20 new amino acids are added per second in eukaryotes and bacteria, respectively. In addition, the high concentration of molecules in the cytosol $(300-400 \mathrm{mg} / \mathrm{ml})$ causes excluded volume effects that enhance the non-native intra- and intermolecular contacts of the newly synthesized polypeptides and thereby increases the risk of misfolding and aggregation

Corresponding author: Deuerling, E. (elke.deuerling@uni-konstanz.de)
[3]. The cellular strategy to safeguard de novo protein folding involves a set of molecular chaperones to prevent such off-pathway reactions [4-8].

Chaperones involved in the de novo folding of cytosolic proteins can be divided into two groups based on their cellular localization (Figure 1). The first group comprises chaperones that dynamically bind to both the ribosome and the emerging polypeptide, thereby controlling early folding steps during translation. Strikingly, cells from different kingdoms of life have evolved diverse types of ribosomeassociated chaperones that work through different structural and mechanistic principles. For instance, bacteria possess the chaperone trigger factor (TF) (Figure 1a), whereas eukaryotes have two different types of ribosome-associated systems that support de novo folding pathways (Figure 1b,c). In yeast, the first system consists of the ribosome-associated complex (RAC) and the Hsp70 chaperone Ssb. RAC is a stable complex formed by the Hsp40 chaperone zuotin (Zuo), and the Hsp70 chaperone Ssz. The second system is the heterodimeric nascent polypeptide-associated complex (NAC) (Figure 1b). Whereas NAC is highly conserved from yeast to humans, mammalian RAC (mRAC) consists of the Zuo homolog MPP11 and an Ssz-like protein Hsp70L1 (Figure 1c) [9] but lacks a ribosome-bound Ssb-like protein. Instead, mammalian cytosolic Hsp70 is recruited to nascent polypeptides via mRAC [10].

The second group of chaperones that acts on newly synthesized proteins mainly comprises the cytosolic chaperones of the Hsp70/40 and Hsp60/10 families, which are present in pro- and eukaryotes, as well as prefoldin, which is present in Archaea and eukaryotes (Figure 1). Both ribosome-associated and cytosolic chaperones cooperate to form a robust network for de novo protein folding. This network is best studied in Escherichia coli. Whereas the Hsp60/10 chaperone system GroEL/GroES is essential under all conditions, cells with individual deletions of the genes encoding TF and the Hsp70 protein DnaK are viable [11-13]. However, simultaneous deletion of genes encoding $\mathrm{TF}$ and DnaK causes cell death at $30^{\circ} \mathrm{C}$ and above [11,12]; this synthetic lethality can be suppressed by GroEL/GroES overexpression, which emphasizes the overlapping substrate pools and chaperone functions of these three systems $[14,15]$.

\section{Trigger factor}

TF is by far the best-understood ribosome-associated chaperone and its role in cotranslational protein folding is well 


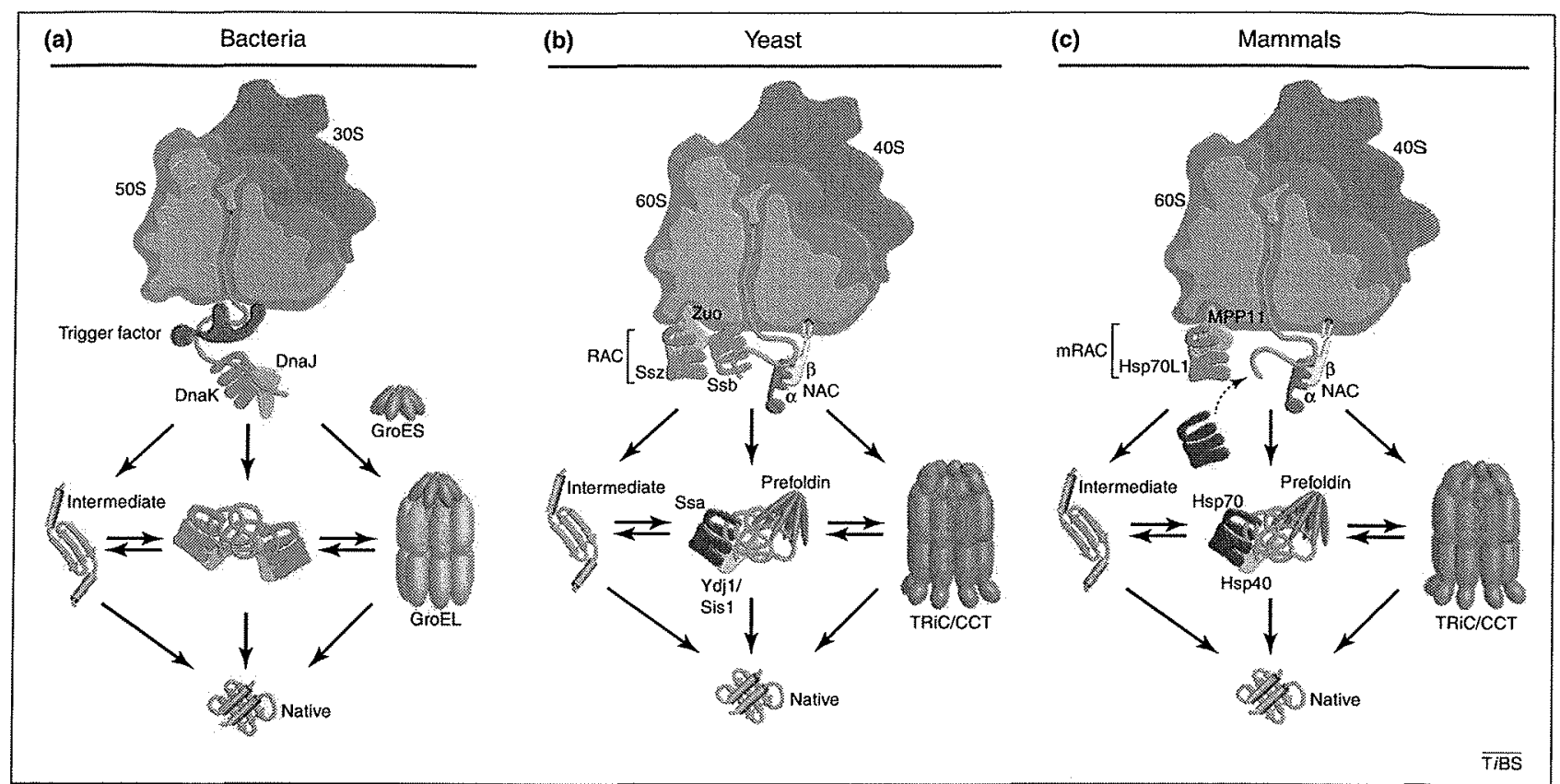

Figure 1. Chaperone networks involved in de novo protein folding. The concept of chaperone systems that assist de novo protein folding is conserved across all kingdoms of life. In (a) Escherichia coli, (b) Saccharomyces cerevisiae and (c) mammals, chaperones bind directly to ribosomes (grev) to act on nascent polypeptides (orange). These ribosome-associated chaperones comprise trigger factor in bacteria and the eukaryotic $\alpha-\beta$ heterodimer nascent polypeptide-associated complex (NAC). In addition, the heat shock protein (Hsp)70/40-based systems Zuo/Ssz/Ssb and MPP11/Hsp70L1 localize to ribosomes in yeast and mammals, respectively. The ribosome-associated complex (RAC) consists of Ssz and zuotin (Zuo) in yeast [48]. MPP11 and Hsp70L1 form mammalian RAC (mRAC). Ssb homologs are restricted to fungi, whereas mammalian RAC recruits cytosolic Hsp70 to nascent polypeptides [10]. Members of the Hsp70/40 and Hsp60/10 chaperone families act downstream of ribosome-associated chaperones on a subset of newly synthesized proteins that require further folding assistance. These include the DnaK/DnaJ and GroEL/GroES chaperone systems in bacteria, and Ssa-Ydj1/Sis1 and the chaperonin TRiC/CCT in yeast $[4,11,13,30,64]$. Hsp $70-H s p 40$ chaperones and TRiC/CCT also interact with newly synthesized proteins in mammalian cells. Specialized chaperones, such as prefoldin, participate in de novo folding of certain substrates $[65,66]$.

characterized. This chaperone is only found in bacteria and chloroplasts. It consists of three domains: an $\mathrm{N}$ domain, a peptidyl-prolyl cis-trans isomerase (PPIase) domain and a $\mathrm{C}$ domain. TF adopts an extended three-dimensional conformation with the $\mathrm{C}$ domain positioned in the center of the molecule (Figure 2a,b) $[16,17]$. The $\mathrm{N}$ domain uses a conserved signature motif to mediate binding to the ribosome via ribosomal protein (r-protein) L23 at the rim of the ribosomal tunnel exit (Figure $2 \mathrm{~b}, \mathrm{~d}$ ). The TF $\mathrm{N}$ domain, together with the $\mathrm{C}$ domain, forms an open cavity with two protruding arms [16-18]. The PPIase domain is located at the distal end of TF. It has been suggested that this domain prolongs the residence time of TF on nascent chains and thereby may contribute to delay cotranslational folding (discussed later). However, the PPIase activity of TF is not essential in vivo and TF variants lacking the isomerase function show a chaperone activity that is comparable to wild type TF $[15,19,20]$.

TF exists in a monomer-dimer equilibrium. In its monomeric state, TF associates transiently with ribosomes and hunches over the ribosomal tunnel, facing its central cavity towards the tunnel exit. Therefore, TF is ideally positioned to capture emerging chains (Figure $2 \mathrm{~d}$ ). TF has multiple binding sites for substrates throughout its interior, which is lined by several hydrophobic side chains that can provide multiple hydrophobic contact sites for an unfolded polypeptide chain (Figure 2c) $[16,21,22]$. Indeed, the residence time of TF on translating ribosomes (between 10 and $50 \mathrm{~s}$ ) correlates with the hydrophobicity of the nascent polypeptide [23]. In addition to the hydrophobic contacts, the cavity also exposes hydrophilic side chains; these might be involved in electrostatic contacts to substrates. Accordingly, it has been shown that TF binds to the folded and positively charged r-protein S7 [24,25]. Thus, the substrate interface of TF is versatile, using different types of interactions, which could explain its broad substrate spectrum (discussed later).

TF accommodates the substrate in its interior, which provides a protective environment to prevent proteins from. aggregating or being degraded (Figure 2d) [26,27]. In addition, ribosome-bound TF can prevent premature and incorrect folding of proteins during synthesis. For example, it retards cotranslational folding of recombinantly expressed firefly luciferase in $E$. coli cells, thereby enhancing the total yield of active luciferase $[23,28]$. By contrast, it is unclear whether TF can also promote cotranslational folding processes. The local accumulation of foldingcompetent polypeptide segments within the TF cavity may favor the formation of key contacts in the nascent chain and thus drive initial folding steps. On the basis of its crystal structure, TF can accommodate entire protein domains or even small proteins (with a size up to 130 aa) in its central cavity [16]. Indeed, it has been shown that purified TF stimulates the refolding of denatured glyceraldehyde 3-phosphate dehydrogenase to its native state in the absence of ribosomes [29]. However, whether this also holds true for the folding of nascent polypeptide chains awaits experimental proof. 


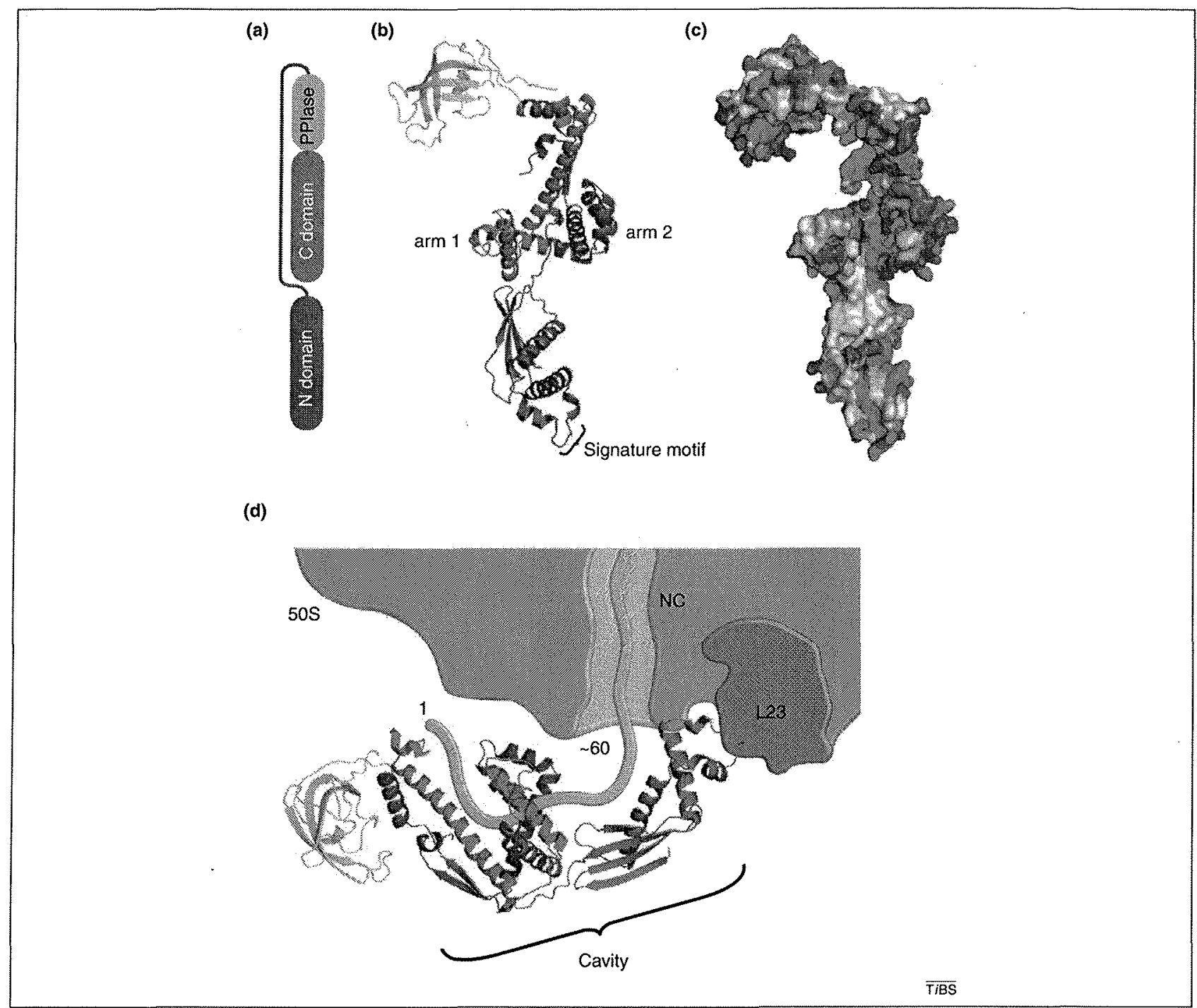

Figure 2. Trigger factor (TF) from Escherichia coli. (a) Domain organization of TF. (b) Ribbon diagram of the crystal structure of TF (PDB 1W26). The $N$ domain (red) contains the TF signature motif (GFRXGXXP) in a loop region between two $\alpha$-helices and is connected to the peptidyl-prolyl cis-trans isomerase (PPlase) domain (green) via an extended linker. The $\mathrm{C}$ domain (blue) is located in the center of the molecule and forms two arm-like protrusions. The $\mathrm{N}$ domain and both arms of the $\mathrm{C}$ domain together form a cavity for nascent polypeptide chains. (c) Surface charge distribution of TF for the same orientation as in (b). Positively and negatively charged residues are shown in blue and red, respectively. (d) Structural model of TF bound to the large ribosomal subunit (grey) based on [16]. The main contact between TF [colors as in (a) and (b)] and the large ribosomal subunit (grey) involves the signature motif in the $\mathrm{N}$ domain of TF and the ribosomal protein L23 (dark grey). In vivo, TF is preferentially recruited to translating ribosomes carrying nascent chains (orange) longer than 100 amino acids (aa), which have $\sim 60$ aa exposed outside the ribosomal tunnel. TF structures were prepared using PyMOL (DeLano Scientific LLC) based on [16].

What are the substrates of ribosome-bound TF?

The first attempts to uncover TF substrates identified proteins that misfold and aggregate in the absence of TF and the cooperating DnaK (Hsp70) system [11,13]. Although deletion of TF alone does not cause protein aggregation, an additional reduction in DnaK levels results in substantial protein misfolding and aggregation. More than 300 different aggregation-prone protein species were found in TF- and DnaK-deficient cells. The proteins identified are involved in many different cellular processes, range in size from 16 to $140 \mathrm{kDa}$, and are specifically enriched in large $(>40 \mathrm{kDa})$ multidomain proteins. Moreover, a proteomewide analysis of the GroEL interactome revealed that $\sim 250$ cytosolic proteins interact with GroEL during folding, but that this number increases substantially in cells lacking TF and DnaK [30]. The data suggest a strong overlap of substrate pools for TF, DnaK and GroEL, which explains the robustness of the chaperone network for newly synthesized proteins in $E$. coli.

A recent study using selective ribosome profiling provided a global analysis of the nascent interactome of TF for the first time [31]. The technique combines crosslinking of endogenously synthesized tagged TF to nascent polypeptides and affinity purification of ribosome-TF complexes and subsequent identification of the mRNA that is being read by TF-bound ribosomes. This elegant approach revealed new fundamental features of the cotranslational activity of TF. The first surprise was that recruitment of TF 
to translating ribosomes is delayed in vivo until nascent peptides reach a length of $\sim 100$ aa. This means that, assuming that $30-40$ aa of the nascent chains are buried in the ribosomal tunnel, TF must bind to emerging peptides only once they have exposed at least $60-70$ aa outside the ribosome (Figure 2d). The finding that TF does not interact with short nascent polypeptides in vivo contradicts in vitro crosslinking studies that suggest that TF contacts peptides immediately on their exit from the ribosomal tunnel [21]. This apparent contradiction is resolved by taking into account that additional factors interact with nascent proteins in vivo. The initial exclusion of TF, which only correlates with the length of the polypeptides, provides a time window to allow processing enzymes to access nascent proteins [31]. These enzymes are required, for example, to remove the formyl moiety and the initiator methionine from the $\mathrm{N}$ termini of nascent polypeptide chains. The second important finding from the ribosome profiling study is that TF interacts with all newly synthesized proteins, except for those inserted into the cytoplasmic membrane via SRP targeting. By contrast, nascent outer membrane $\beta$-barrel proteins (Omps) were among the strongest TF interactors during their synthesis on ribosomes. This suggests that the chaperone activity of $E$. coli TF is particularly important for keeping Omps in a translocation-competent conformation, so that they can be efficiently exported by the Sec machinery (Figure 5a) [31]. It should be mentioned that this finding is in agreement with pioneering studies by Wickner and coworkers, who initially identified TF in a reconstituted in vitro translocation experiment as a cytosolic component that maintains proOmpA in a transport-competent conformation for delivery into inside-out membrane vesicles [32]. However, because TF is also evolutionarily conserved in Gram-positive bacteria that lack an outer membrane, it is obvious that TF is required to assist in the folding of many cytosolic proteins as well.

\section{More than one function for TF?}

Without doubt, $\mathrm{TF}$ is a chaperone for nascent polypeptides, but there might be another function for the nonribosomal dimeric form of TF in the cytosol (Figure 5a). MartinezHackert and Hendrickson discovered that TF also associates with proteins that are normally assembled into large complexes [25]. More than 60 different full-length proteins, including many $r$-proteins such as the $30 \mathrm{~S}$ protein $\mathrm{S} 7$, were copurified with recombinant TF from $E$. coli lysates. Subsequent structural analysis of TF from Thermotoga maritima (TF-Tm) in a 2:2 stoichiometric complex with the rprotein $57-T m$ revealed that TF-Tm is able to bind and stabilize almost natively folded S7 proteins. The structural data indicate that TF-Tm masks large areas of the surface of $\mathrm{S7}$, which are substantially buried on assembly into the $30 \mathrm{~S}$ ribosomal subunit. Therefore, it was suggested that TF might act as an assembly factor for large protein complexes and ribosomes (Figure 5a). However, the physiological importance of an assembly function of TF remains unclear because cells lacking TF show only a very mild defect in ribosome biogenesis at elevated temperatures. Nevertheless, it is an attractive hypothesis that TF has more than one function. Considering that TF is present in a 1-2 molar excess over ribosomes [33] and preferentially associates with translating ribosomes after synthesis of the first 100 aa, enough TF is available to serve both activities: cotranslational chaperoning of newly made proteins and posttranslational assembly support for large complexes.

\section{Eukaryotic ribosome-associated chaperones}

In eukaryotes, a Hsp70/40 system and NAC are assumed to function in cotranslational protein folding (Figure $1 \mathrm{~b}, \mathrm{c}$ ). However, their mechanisms of action and structures are barely characterized. As with bacterial TF, these eukaryotic factors are abundant cellular proteins that can cycle on and off the ribosome and may have additional functions in the cytosol or nucleus. Moreover, the presence of nascent polypeptides enhances ribosome association of these chaperones, although no kinetic data exist so far [34].

\section{NAC}

NAC has been described as the initial factor that interacts with nascent polypeptides as they emerge from ribosomes and prevents them from forming incorrect interactions [35]. The complex is widely conserved from Archaea to man. However, Archaea only have a homodimeric NAC formed by two $\alpha$-subunits, whereas yeast and higher eukaryotes form an $\alpha-\beta$ heterodimer (Figures $1 \mathrm{~b}, \mathrm{c}$ and 3). Crystal structures of truncated NAC variants from archaeal and human complexes suggest that homo- and heterodimers of NAC are formed via their homologous sixstranded $\beta$-barrel-like NAC domains, one of which is present in $\alpha$ - and $\beta$-NAC subunits, respectively (Figure $3 \mathrm{~b}$ ). In contrast to $\beta-\mathrm{NAC}$, the $\alpha-\mathrm{NAC}$ subunit contains a C-terminal ubiquitin-associated (UBA) domain [36-38], suggesting that there could be different functions for the two subunits $[4,36]$; however, the role of the UBA domain is unknown.

No structural information for the NAC ribosome-binding element exists. However, it is known that the $\mathrm{N}$ terminus of the $\beta$-NAC subunit of eukaryotic NAC harbors a conserved ribosome-binding motif (Figure $3 \mathrm{a}$ ) and ribosome binding might involve the predicted adjacent $\alpha$-helical element as well. Crosslinking data suggest that there may be multiple anchor sites for NAC on ribosomes, including the r-protein Rpl25, the yeast homolog of bacterial L23 [39-41]. The conserved r-protein L23/Rpl25 serves as a multifunctional docking site and coordinates other ribosome-associated factors such as TF (in bacteria), SRP and the Sec translocation pore [7]. Importantly, ribosome binding seems to be a prerequisite for NAC interaction with nascent polypeptides, because amino acid substitutions in the ribosome-binding motif of $\beta$-NAC that diminish ribosome association also prevent crosslinking to nascent chains in vitro $[35,39]$. Although $\beta-N A C$ mediates association of the heterodimeric complex with ribosomes, both NAC subunits contact nascent polypeptides. However, the substrate binding sites in the individual NAC subunits are not yet identified.

Multiple functions have been described for NAC, including roles as a transcription activator, a mediator of ER stress and a triage factor for transport to mitochondria and the ER [42]. However, most of these potential NAC functions still lack compelling in vivo evidence. The observation 


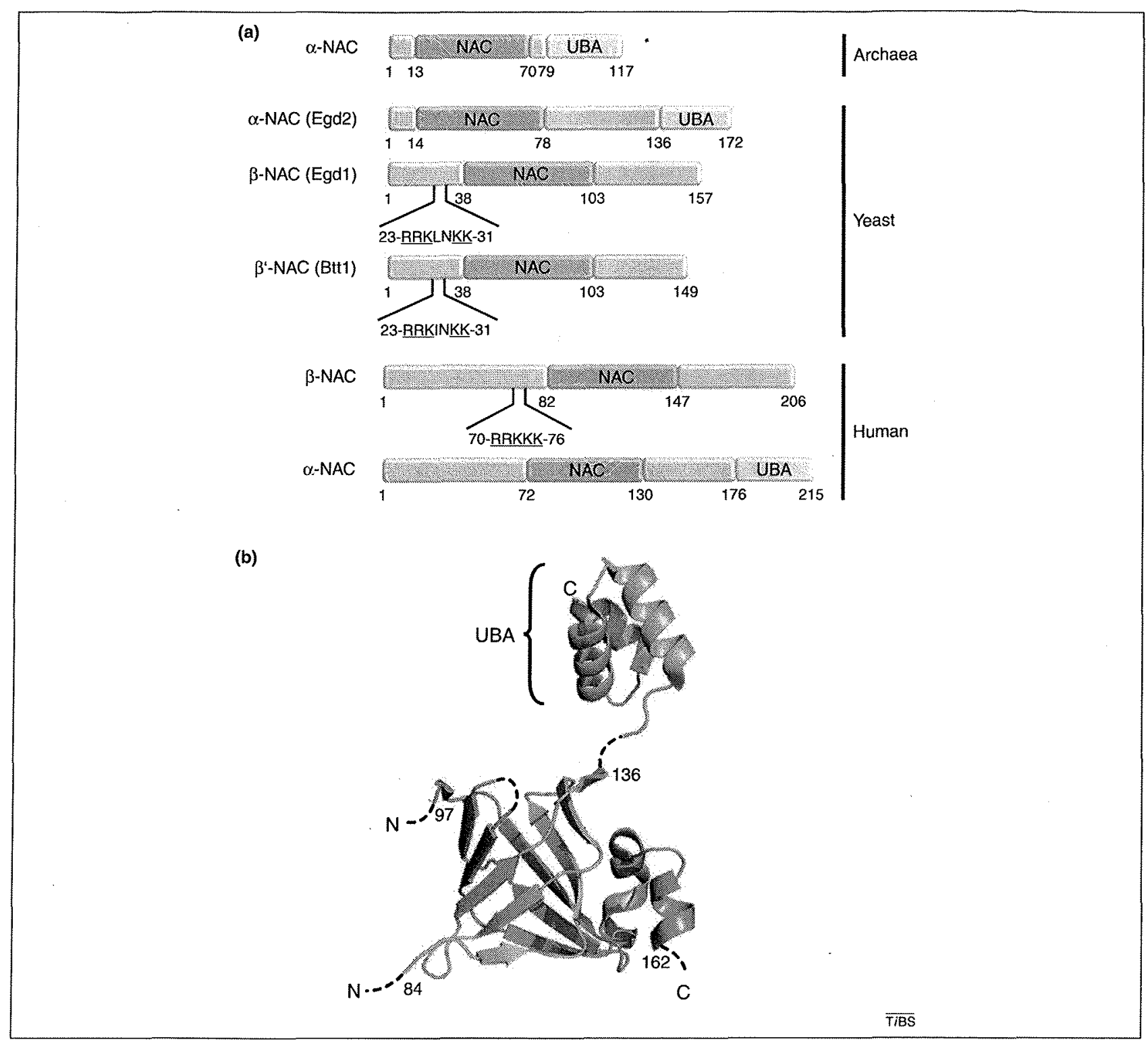

Figure 3. The nascent polypeptide-associated complex (NAC). (a) Schematic representation of the conserved domains of archaeal, yeast (Saccharomyces cerevisiae) and human NAC. The conserved NAC domains (green) and the ubiquitin-associated (UBA) domain (vellow) of $\alpha-N A C$ are indicated. The conserved NAC signature motif, which mediates ribosome binding [39], is indicated for the yeast and human $\beta-N A C$ subunits. (b) Structural model of NAC. $\alpha-N A C$ (blue) and $\beta-N A C$ (orange) form a stable heterodimer via the NAC domains. The UBA domain is derived from the crystal structure of archaeal NAC (PDB 1TR8) [36] and was modeled on the human NAC domain heterodimer [PDB 3LKX, aa 84-136 of $\alpha-N A C$ (NACA) and aa 97-162 of $\beta-N A C$ (BTF3) isoform A] [37]. Broken lines indicate unresolved parts of the molecule. The figure was generated using PyMOL (DeLano Scientific LLC).

that NAC quantitatively associates with ribosomes in $1: 1$ stoichiometry, crosslinks to nascent proteins and protects them from proteolysis suggests that this complex might be a component of the chaperone network in eukaryotes that is dedicated to support de novo folding processes [7,34]. Moreover, loss of NAC function in yeast results in no detectable phenotype; however, the absence of NAC significantly exacerbates the growth defects of cells lacking the ribosome-associated Hsp70 homolog Ssb at $30^{\circ} \mathrm{C}$ and strongly enhances aggregation of newly synthesized proteins [43]. This suggests that NAC is genetically and functionally connected to the second ribosome-attached chaperone system. Because of the robustness of the chaperone network, loss of NAC alone might be compensated for in yeast. By contrast, NAC mutations in mice, fruit flies and Caenorhabditis elegans cause developmental defects and early embryonic lethality, suggesting additional irreplaceable NAC functions in higher eukaryotes [44-46].

A study by Frydman and colleagues further supports the hypothesis that NAC acts as an integral component of the chaperone network that promotes folding of newly synthesized proteins. They systematically identified cotranslational substrates of NAC via the mRNAs associated with ribosome-NAC complexes [47]. The data revealed that NAC binds to virtually all translating ribosomes and contacts every nascent polypeptide made in 
yeast. In contrast to higher eukaryotes, yeast has three NAC subunits (Figure 3a): two B-NAC (Egd1 and Btt1) and one $\alpha$-NAC (Egd2), which give rise to different NAC homo- and heterodimers with different substrate specificities [45,47]. Btt1-subunit homo- and heterodimers predominantly bind to ribosomes that translate mitochondrial or ribosomal proteins. By contrast, Egd1 and Egd2, which form the most abundant NAC heterodimer in yeast, show a preference for ribosomes that translate metabolic enzymes and secretory and membrane proteins [47]. By comparing the interactome of NAC (Egd1/Egd2) with that of SRP, Frydman and coworkers found that a subset of nascent secretory proteins interacts with both factors. Although yeast cells lacking NAC do not have pronounced impairments in targeting of proteins to their appropriate compartments, some secreted polypeptides require NAC to efficiently interact with SRP, whereas others are prevented from interacting with SRP when NAC is present [47]. Therefore, yeast NAC appears to act as a triage factor for certain secretory nascent polypeptides that are targeted by SRP to the Sec translocation pore (Figure $5 b$ ).
The ribosome-associated Hsp70/40 system

The ribosome-associated Hsp70/40 chaperone system is only found in eukaryotes. The conserved core element is the stable heterodimeric RAC. In Saccharomyces cerevisiae, this system is complemented by another ribosomeassociated Hsp70, Ssb, whereas in higher eukaryotes cytosolic Hsp70 acts together with the ribosome-bound mRAC system (Figures 1 b,c and 4) $[9,10,48,49]$.

There are no structural data for RAC, but hydrogendeuterium exchange experiments, combined with mutational analysis, gave insights into the overall architecture of the complex [50]. The $\mathrm{N}$ terminus of Zuo stably binds to the Hsp70 protein Ssz, mainly via contacts with the Cterminal substrate-binding domain (SBD) and some contacts with the N-terminal nucleotide-binding domain (NBD) of Ssz (Figure 4a). Furthermore, Zuo has a J domain that stimulates the ATPase activity of Ssb and a charged region that allows $\mathrm{RAC}$ to bind the ribosome near the ribosomal protein $\mathrm{Rpl31}$ at the ribosomal tunnel exit [51]. In contrast to canonical J-domain proteins, Zuo requires stable interaction with $\mathrm{Ssz}$ to act as a co-chaperone for $\mathrm{Ssb}[48,52]$. Thus far, RAC association with nascent

(a)
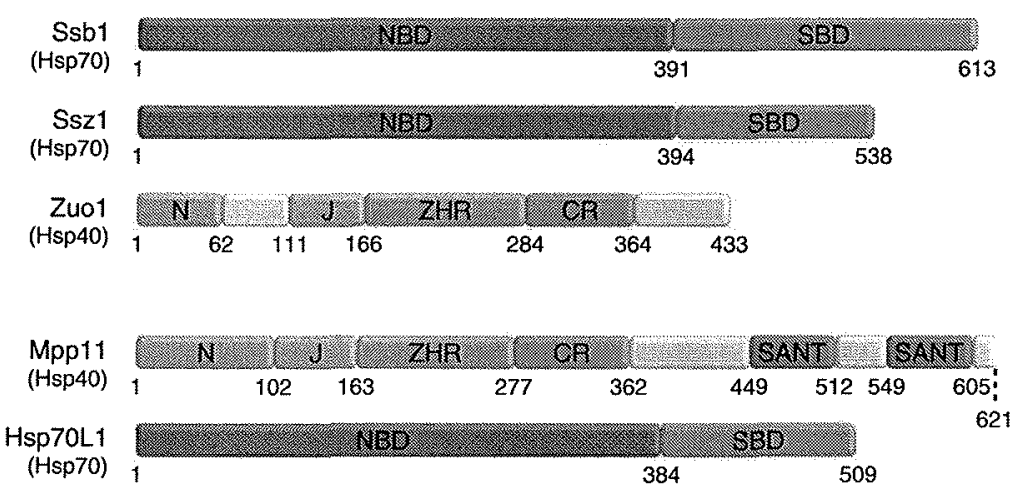

(b)

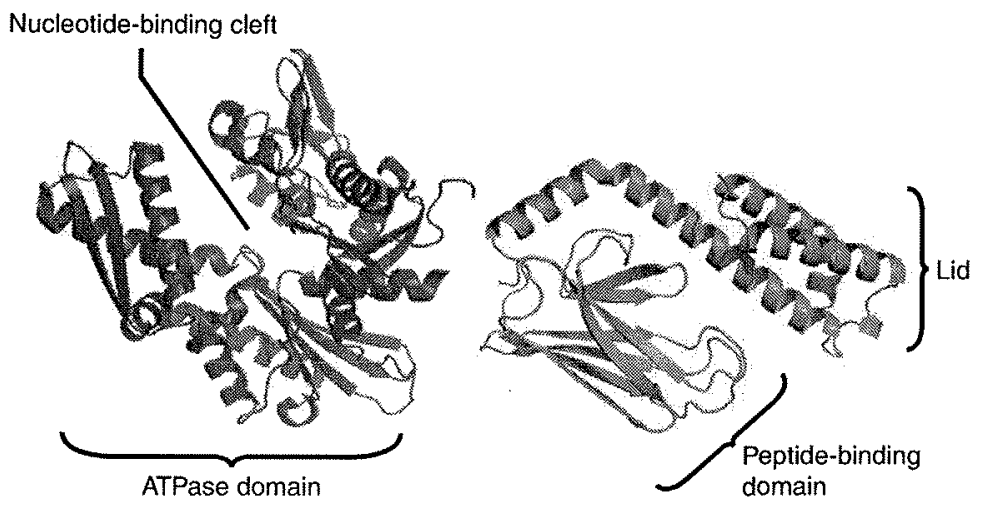

$\overline{\text { TiBS }}$

Figure 4. RAC Ssb from yeast and its mammalian homolog mRAC. (a) Schematic representation of the conserved domains of yeast Ssb and RAC (Ssz and Zuo) and mammalian RAC (MPP11 and Hsp70L1). NBD, nucleotide-binding domain; SBD, substrate-binding domain; N, N-terminal domain; J, J domain; ZHR, Zuo homology region; CR, charged region; SANT, SANT domain. (b) Structural model of Ssb1 from S. cerevisiae. The ATPase domain (PDB 3GL1) is shown in blue. A model of the Cterminal substrate-binding domain (green) was generated using I-TASSER protein structure and function prediction [67]. The figure was generated using PyMOL (DeLano Scientific LLC). 
polypeptides has never been shown, but it is essential for stimulating the binding of Ssb to nascent substrates.

Zuo homologs also exist in mammals (MPP11), but have some variations in their domain composition, including two additional SANT-like domains at the C-terminal end of the chaperone (Figure 4a) [9,49]. SANT domains are normally involved in DNA binding and chromatin remodeling [53]; however, their role in MPP11 is unknown. The Zuo homologs share the $\mathrm{J}$ domain and the charged region for ribosome binding. Thus, they are likely to function in a similar manner on ribosomes. This assumption is supported by the finding that human MPP11 can complement the phenotype caused by deletion of the gene encoding Zuo in yeast. Hsp70L1 was identified as the RAC partner (analogous to Ssz) for human MPP11 (Figures $1 \mathrm{c}$ and, 4a) $[9,49]$.

$\mathrm{Ssb}$ is only found in fungal species, such as yeast. Its structure is typical of Hsp70 proteins: it contains an $\mathrm{N}$-terminal ATPase domain connected via a linker to the C-terminal SBD (Figure 4b). Similar to classical cytosolic Hsp70 s, the Ssb reaction cycle is driven by co-chaperones. These include RAC, which stimulates ATP hydrolysis for tight substrate binding, and nucleotide exchange factors such as Sse1, SIn 1 and Fes1, which exchange ADP for ATP, thus triggering substrate release [54]. Ssb associates with ribosomes at the ribosomal exit site in a 1:1 stoichiometry that is independent of RAC. Yeast cells lacking Ssb, RAC or both have a very similar phenotype, which includes sensitivity to high salt concentrations, low temperature and translation inhibitory drugs [54,55]. This suggests that these components form a functional unit on ribosomes.

As demonstrated recently, the loss of Ssb causes misfolding of newly synthesized proteins and the accumulation of protein aggregates [43]. Interestingly, protein aggregation was significantly enhanced in cells lacking NAC and Ssb simultaneously, although the pattern of aggregated proteins remained similar, suggesting overlapping substrate pools for the two ribosome-associated systems. Mass spectrometry analysis identified mainly $\mathbf{r}$ proteins in the insoluble fraction of yeast cells lacking Ssb and NAC [43]. Strikingly, 52 out of 78 r-proteins were identified in the aggregates. This suggests that r-proteins are among the major substrates for RAC-Ssb and NAC.

\section{Do RAC-Ssb and NAC play a role in ribosome} biogenesis and the regulation of protein synthesis? Cells lacking Ssb or both Ssb and NAC not only accumulate protein aggregates but also have strongly decreased levels of the $40 \mathrm{~S}$ and $60 \mathrm{~S}$ ribosomal subunits and reduced translational activity $[43,56,57]$. Moreover, combined deletion of genes encoding either Ssb or Zuo and $\mathrm{Jjj} 1$, a chaperone specific for maturation of the $60 \mathrm{~S}$ ribosomal subunit, caused synthetic lethality in yeast. Several defects in ribosome biogenesis were discovered in cells lacking components of the RAC-Ssb and NAC systems. For instance, accumulation of a GFP-labeled r-protein in the nucleus revealed defects in the assembly and export of pre-60S subunits into the cytoplasm [43,56,57]. In addition, microarray analysis demonstrated that $\mathrm{Ssb}$ and Zuo influence pre-18S and pre-25S rRNA processing in the nucleus
$[43,56,57]$. These data strongly support a role for $\mathrm{RAC}-$ $\mathrm{Ssb}$ and NAC in ribosome assembly. This function is consistent with the suggestion that Zuo, Ssb and NAC cycle between the cytoplasm and the nucleus, and with the observed transcriptional coregulation of $\mathrm{Ssb}$ and $\mathrm{r}$ proteins [42,56,58,59].

How these chaperones function in ribosome biogenesis is an open question. Two models are plausible that are not mutually exclusive (Figure 5b). The first possibility is that the chaperones could bind to r-proteins during translation, remain bound to their substrates after synthesis, accompany $r$-proteins into the nucleus, and then release them directly onto rRNA for assembly. This is feasible, because many $r$-proteins are highly abundant, have complex conformations and only attain their correct structure on assembly into ribosomal particles. The second possibility is that these chaperones actively promote ribosomal maturation steps in the nucleus. This assumption is supported by the finding that Zuo interacts with nuclear ribosome precursors [56]. Given the complexity of the ribosome assembly process, much effort needs to be put into understanding the exact mechanisms and functions of these chaperones.

\section{Are NAC and RAC-Ssb systems involved in managing protein aggregation?}

An elegant screen for proteins that interact with different artificial aggregation-prone $\beta$-sheet proteins revealed that NAC associates with insoluble amyloid-like fibers in mammalian cells [60]. In addition, yeast Ssb associates with insoluble polyglutamine repeat proteins in vivo [61] and modulates the formation and toxicity of the Sup35 prion [62]. Although the physiological importance of these findings is not clear, an attractive hypothesis is that the NAC and RAC-Ssb systems not only cycle between different cellular compartments but also associate with different macromolecular assemblies in the cytosol (ribosomes and protein aggregates) to exert versatile chaperoning functions (Figure 5b). Finally, it should be mentioned that a recent study reported yet another ribosome-independent function of $\mathrm{Ssb}$. This study showed that $\mathrm{Ssb}$ regulates the phosphorylation level of Snf1 protein kinase, thereby allowing an appropriate response to changing glucose concentrations [63].

\section{Emerging concepts of functional versatility}

The myriad of recently accumulated data on the in vivo roles of ribosome-associated chaperones from pro- and eukaryotic kingdoms suggests that they are versatile and vital elements of the chaperone network and perform more than one function. There is a striking functional analogy among these diverse chaperones. They cotranslationally support de novo folding and also act through unknown mechanisms to regulate ribosome biogenesis and thereby control protein synthesis. The latter is intriguing, because it allows these chaperones to regulate the influx of all new proteins into the cellular proteome beyond just those that require their assistance for folding. In addition to their ribosome-associated cotranslational roles, $\mathrm{TF}$ and NAC are important for modulating transport processes across membranes, and Ssb and NAC associate with protein aggregates. Thus, ribosome-associated chaperones 


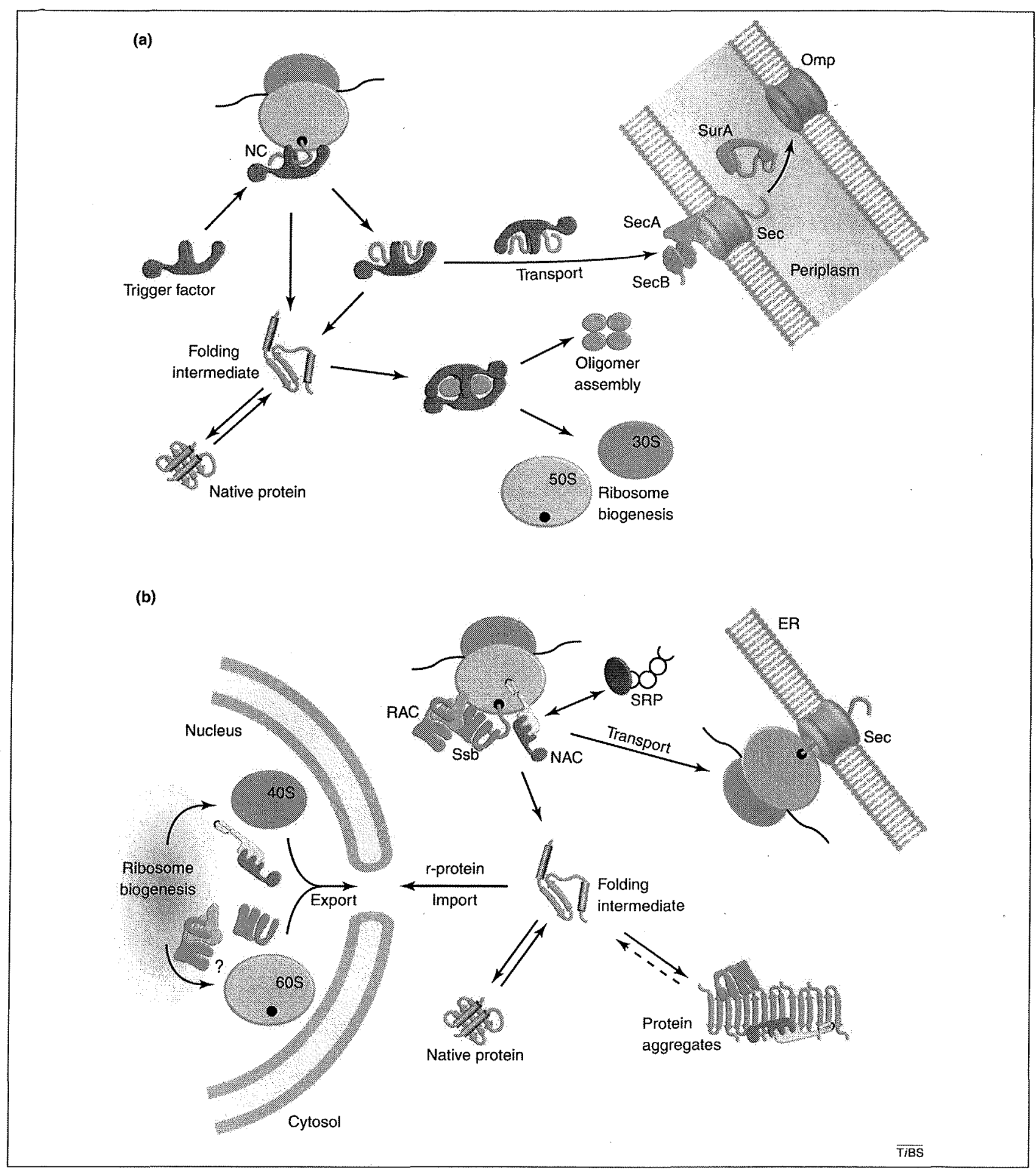

Figure 5. Ribosome-associated chaperones are functionally versatile and act in diverse cellular processes. (a) On the ribosome, TF protects cytosolic nascent polypeptides (orange) from aggregation, proteolysis and premature de novo folding [68]. In E. coli, TF strongly binds to outer membrane proteins (Omps) and supports targeting of translocation-competent $\mathrm{Omp}$ precursors to the cytoplasmic membrane for post-translational translocation through the secretion (Sec) transiocation pore into the periplasm. This pathway probably involves the motor ATPase SecA and the translocation-dedicated chaperone SecB. In the periplasm, SurA, a chaperone with structural features similar to TF, assists Omp transport and membrane insertion [31]. In addition, nonribosomal TF facilitates the assembly of protein oligomers and the incorporation of ribosomal proteins into ribosome subunit precursors [25]. (b) In yeast, NAC and RAC-Ssb bind to ribosomes and interact with nascent polypeptide chains to assist de novo folding $[4,11,13,30,64]$. NAC may also modulate the interaction of SRP with nascent polypeptides and thus contribute to cotranslational targeting of secretory proteins to the ER membrane [47]. NAC (human) and Ssb were both found in association with amyloid-like aggregates $[60,61]$, indicating a possible function in protein aggregation, disaggregation or aggregate clearance. In addition, RAC-Ssb and NAC have functions in the nucleus, including ribosome biogenesis [43,56]. 
are potent modulators of protein synthesis, folding, assembly and transport. Therefore, they represent key regulators of cellular proteostasis. A future challenge will be to dissect their individual roles in this complex network of interconnected biological processes that maintains proteostasis.

\section{Acknowledgments}

We apologize that we could not discuss all aspects of de novo folding and chaperone functions in depth and apologize to all our colleagues whose recent research was not or only very briefly discussed or not cited because of space constraints. We thank the members of the Deuerling laboratory for critically reading and discussing the manuscript. Research in our laboratory is supported by the SFB 969 of the German Science Foundation (DFG) and the Konstanz Research School Chemical Biology.

\section{References}

1 Kramer, G. et al. (2009) The ribosome as a platform for co-translational processing, folding and targeting of newly synthesized proteins. Nat. Struct. Mol. Biol. 16, 589-597

2 Nissen, P. et al. (2000) The structural basis of ribosome activity in peptide bond synthesis. Science $289,920-930$

3 Ellis, R.J. and Minton, A.P. (2006) Protein aggregation in crowded environments. Biol. Chem. 387, 485-497

4 Hartl, F.U. et al. (2011) Molecular chaperones in protein folding and proteostasis. Nature $475,324-332$

5 Buchberger, A. et al. (2010) Protein quality control in the cytosol and the endoplasmic reticulum: brothers in arms. Mol. Cell 40, 238-252

6 Frydman, J. (2001) Folding of newly translated proteins in vivo: the role of molecular chaperones. Annu. Rev. Biochem. 70, 603-647

7 Wegrzyn, R.D. and Deuerling, E. (2005) Molecular guardians for newborn proteins: ribosome-associated chaperones and their role in protein folding. Cell. Mol. Life Sci. 62, 2727-2738

8 Deuerling, E. and Bukau, B. (2004) Chaperone-assisted folding of newly synthesized proteins in the cytosol. Crit. Rev. Biochem. Mol. Biol. 39, 261-277

9 Otto, H. et al. (2005) The chaperones MPP11 and Hsp70L1 form the mammalian ribosome-associated complex. Proc. Natl. Acad. Sci. U.S.A. $102,10064-10069$

10 Jaiswal, H. et al. (2011) The chaperone network connected to human ribosome-associated complex. Mol. Cell. Biol. 31, 1160-1173

11 Deuerling, E. et al. (1999) Trigger factor and DnaK cooperate in folding of newly synthesized proteins. Nature 400,693-696

12 Teter, S.A. et al. (1999) Polypeptide flux through bacterial Hsp70: DnaK cooperates with Trigger Factor in chaperoning nascent chains. Cell 97, 755-765

13 Deuerling, E. et al. (2003) Trigger Factor and DnaK possess overlapping substrate pools and binding specificities. Mol. Microbiol. $47,1317-1328$

14 Vorderwülbecke, S. et al. (2004) Low temperature or GroEL/ES overproduction permits growth of Escherichia coli cells lacking Trigger Factor and DnaK. FEBS Lett. 559, 181-187

15 Genevaux, P. et al. (2004) In vivo analysis of the overlapping functions of DnaK and trigger factor. EMBO Rep. 5, 195-200

16 Ferbitz, L. et al. (2004) Trigger Factor in complex with the ribosome forms a molecular cradle for nascent proteins. Nature $431,590-596$

17 Kramer, G. et al. (2002) L23 protein functions as a chaperone docking site on the ribosome. Nature 419, 171-174

18 Merz, F. et al. (2006) The C-terminal domain of $E$. coli Trigger Factor represents the central module of its chaperone activity. J. Biol. Chem. $281,31963-31971$

19 Kramer, G. et al. (2004) Trigger Factor peptidyl-prolyl cis/trans isomerase activity is not essential for the folding of cytosolic proteins in Escherichia coli. J. Biol. Chem. 279, 14165-14170

20 Gupta, R. et al. (2010) Trigger factor lacking the PPIase domain can enhance the folding of eukaryotic multi-domain proteins in Escherichia coli. FEBS Lett. 584, 3620 -3624

21 Merz, F. et al. (2008) Molecular mechanism and structure of Trigger Factor bound to the translating ribosome. EMBO J.27, 1622-1632

22 Lakshmipathy, S.K et al. (2007) Identification of nascent chain interaction sites on trigger factor. J. Biol. Chem. 282, 12186-12193
23 Kaiser, C.M. et al. (2006) Real-time observation of trigger factor function on translating ribosomes. Nature 444, 455-460

24 Lakshmipathy, S.K. et al. (2010) Versatility of trigger factor interactions with ribosome-nascent chain complexes. $J$. Biol. Chem. $285,27911-27923$

25 Martinez-Hackert, E. and Hendrickson, W.A. (2009) Promiscuous substrate recognition in folding and assembly activities of the trigger factor chaperone. Cell 138, 923-934

26 Hoffmann, A. et al. (2006) Trigger Factor forms a protective shield for nascent polypeptides at the ribosome. J. Biol. Chem. 281, 6539-6545

27 Tomic, S. et al. (2006) Exploring the capacity of trigger factor to function as a shield for ribosome bound polypeptide chains. FEBS Lett. 580, 72-76

28 Agashe, V.R. et al. (2004) Function of trigger factor and DnaK in multidomain protein folding: increase in yield at the expense of folding speed. Cell 117, 199-209

29 Huang, G.C. et al. (2002) Chaperone and antichaperone activities of trigger factor. Eur. J. Biochem. 269, 4516-4523

30 Kerner, M.J. et al. (2005) Proteome-wide analysis of chaperonindependent protein folding in Escherichia coli. Cell 122, 209-220

$31 \mathrm{Oh}$, E. et al. (2011) Selective ribosome profiling reveals the cotranslational chaperone action of trigger factor in vivo. Cell 147, 1295-1308

32 Crooke, E. and Wickner, W. (1987) Trigger factor: a soluble protein that folds pro-OmpA into a membrane-assembly competent form. Proc. Natl. Acad. Sci. U.S.A. 84, 5216-5220

33 Lill, R. et al. (1988) The 'Trigger factor cycle' includes ribosomes, presecretory proteins and the plasma membrane. Cell 54, 10131018

34 Raue, U. et al. (2007) Association of protein biogenesis factors at the yeast ribosomal tunnel exit is affected by the translational status and nascent polypeptide sequence. J. Biol. Chem. 282, 7809-7816

35 Wiedmann, B. et al. (1994) A protein complex required for signalsequence-specific sorting and translocation. Nature 370, 434-440

36 Spreter, T. et al. (2005) The crystal structure of archaeal nascent polypeptide-associated complex (NAC) reveals a unique fold and the presence of a ubiquitin-associated domain. J. Biol. Chem. 280 , $15849-15854$

37 Liu, Y. et al. (2010) The crystal structure of the human nascent polypeptide-associated complex domain reveals a nucleic acidbinding region on the NACA subunit. Biochemistry (Mosc.) 49, 2890-2896

38 Wang, L. et al. (2010) Crystal structures of NAC domains of human nascent polypeptide-associated complex (NAC) and its $\alpha$ NAC subunit. Protein Cell 1, 406-416

39 Wegrzyn, R.D. et al. (2006) A conserved motif is prerequisite for the interaction of NAC with ribosomal protein L23 and nascent chains. $J$. Biol. Chem. 281, 2847-2857

40 Franke, J. et al. (2001) Evidence for a nuclear passage of nascent polypeptide-associated complex subunits in yeast. J. Cell Sci. 114 2641-2648

41 Pech, M. et al. (2010) Dual binding mode of the nascent polypeptideassociated complex reveals a novel universal adapter site on the ribosome. J. Biol. Chem. 285, 19679-19687

42 Rospert, S. et al. (2002) Nascent-polypeptide-assaciated complex. Cell. Mol. Life Sci. 59, 1632 1639

43 Koplin, A. et al. (2010) A dual function for chaperones $\mathrm{Ssb} / \mathrm{RAC}$ and the NAC nascent polypeptide-associated complex on ribosomes. J. Cell Biol. 189, 57-68

44 Markesich, D.C. et al. (2000) Bicaudal encodes the Drosophila beta NAC homolog, a component of the ribosomal translational machinery. Development 127, 559-572

45 Reimann, B. et al. (1999) Initial characterization of the nascent polypeptide-associated complex in yeast. Yeast 15, 397 -407

46 Deng, J.M. and Behringer, R.R. (1995) An insertional mutation in the BTF3 transcription factor gene leads to an early postimplantation lethality in mice. Transgenic Res. 4, 264-269

47 del Alamo, M. et al. (2011) Defining the specificity of cotranslationally acting chaperones by systematic analysis of mRNAs associated with ribosome-nascent chain complexes. PLoS Biol. 9, e1001100

48 Gautschi, M. et al. (2001) RAC, a stable ribosome-associated complex in yeast formed by the DnaK-DnaJ homologs Ssz1p and zuotin. Proc. Natl. Acad. Sci. U.S.A. 98, 3762-3767 
49 Hundley, H.A. et al. (2005) Human Mpp11 J protein: ribosometethered molecular chaperones are ubiquitous. Science $308,1032-1034$

50 Fiaux, J. et al. (2010) Structural analysis of the ribosome-associated complex (RAC) reveals an unusual Hsp70/Hsp40 interaction. J. Biol. Chem. 285, 3227 3234

51 Peisker, K. et al. (2008) Ribosome-associated complex binds to ribosomes in close proximity of Rpl31 at the exit of the polypeptide tunnel in yeast. Mol. Biol. Cell 19, 5279-5288

52 Huang, P. et al. (2005) The Hsp70 Ssz1 modulates the function of the ribosome-associated J-protein Zuo1. Nat. Struct. Mol. Biol. 12, 497-504

53 Boyer, L.A. et al. (2004) The SANT domain: a unique histone-tail binding module? Nat. Rev. Mol. Cell Biol. 5, 158-163

54 Peisker, K. et al. (2010) The ribosome-bound Hsp70 homolog Ssb of Saccharomyces cerevisiae. Biochim. Biophys. Acta 1803, 662-672

55 Craig, E.A. et al. (2003) Ribosome-tethered molecular chaperones: the first line of defense against protein misfolding? Curr. Opin. Microbiol. 6, 157-162

56 Albanese, V. et al. (2010) A ribsome-anchored chaperone network that facilitates eukaryotic ribosome biogenesis. J. Cell Biol. 189, 69-81

57 Nelson, R.J. et al. (1992) The translation machinery and $70 \mathrm{kDa}$ heat shock protein cooperate in protein synthesis. Cell 71, 97-105

58 Shulga, N. et al. (1999) A nuclear export signal prevents Saccharomyces cerevisiae Hsp70 Ssb1p from stimulating nuclear localization signal-directed nuclear transport. J. Biol. Chem. 274, 16501-16507
59 Lopez, N.etal. (1999) SSB, encoding a ribosome-associated chaperone, is coordinately regulated with ribosomal protein genes. $J$. Bacteriol $181,3136-3143$

60 Olzscha, H. et al. (2011) Amyloid-like aggregates sequeste numerous metastable proteins with essential cellular functions. Cell 144, 67-78

61 Wang, Y. et al. (2009) Abnormal proteins can form aggresome in yeast: aggresome-targeting signals and components of the machinery. FASEB J. 23, 451-463

62 Chernoff, Y.O. et al. (1999) Evidence for a protein mutator in yeast: role of the Hsp70-related chaperone Ssb in formation, stability, and toxicity of the [PSI] prion. Mol. Cell. Biol. 19, 8103-8112

63 von Plehwe, U. et al. (2009) The Hsp70 homolog Ssb is essential for glucose sensing via the SNF1 kinase network. Genes Dev. 23 , 2102-2115

64 Bukau, B. et al. (2000) Getting newly synthesized proteins into shape. Cell 101, 119-122

65 Hansen, W.J. et al. (1999) Prefoldin-nascent chain complexes in the folding of cytoskeletal proteins. J. Cell Biol. 145, 265-277

66 Hansen, W.J.et al. (1994) Complex environment of nascent polypeptide chains. J. Biol. Chem. 169, 26610-26613

67 Zhang, Y. (2008) I-TASSER server for protein 3D structure prediction. BMC Bioinform. 9,40

68 Hoffmann, A. et al. (2010) Structure and function of the molecular chaperone Trigger Factor. Biochim. Biophys. Acta 1803, 650-661 